\title{
INFINITY NORM MEASUREMENT OF SENSITIVITY FUNCTION BASED ON LIMIT CYCLES IN A CLOSED-LOOP EXPERIMENT
}

\author{
D. Garcia, A. Karimi, R. Longchamp \\ Laboratoire d'Automatique, École Polytechnique Fédérale de Lausanne (EPFL), 1015 Lausanne, Switzerland \\ e-mail: daniel.garciadepfl.ch, alireza.karimidepfl.ch, roland.longchampdepfl.ch
}

Keywords: Describing function, limit cycles, nonlinear feedback test, frequency domain identification, stability analysis

\begin{abstract}
This paper proposes a new method for the measurement of the infinity norm of the sensitivity functions of linear systems. A nonlinear feedback structure is derived from existing relay experiments. The proposed scheme may produce a limit cycle at a frequency where the sensitivity function of a given system achieves a selected magnitude. Necessary and sufficient conditions for the occurrence of the limit cycles are discussed using the describing function method. If the magnitude is chosen larger than the sensitivity infinity norm, the strictly passivity of the scheme can be demonstrated. Based on these analyses, a simple procedure is then proposed to identify the infinity norm of the sensitivity function for a large class of processes. Simulation results show the effectiveness and simplicity of the proposed method.
\end{abstract}

\section{Introduction}

PID controller is the most dominating form of feedback for industrial plants. Nevertheless many of them show poor performances, due among others to inadequate tuning of their parameters. Therefore, several different automatic tuning procedures with varying objectives and complexity are desirable. Autotuning methods operate usually as follows: Some important characteristics of the plant are first measured by simple experiments, which are then used to design or tune the controller parameters. In this perspective, the measurement of robustness margins can reveal a great interest, because these margins and the frequencies at which they occur are crucial for the determination of control system stability and performance. The identified points of the frequency response can be used for obtaining a process model that approximate well the plant in this important frequency region [10]. They can also be employed to adjust the corresponding robustness margins to the desired values and then to verify if the desired robustness specifications are effectively satisfied $[6,7,8]$.

Some attention has been given to the measurement of robustness margins, in particular with the relay method. This technique is well known to identify interesting points on the frequency response of a transfer function. The standard method
[5], based on a simple relay feedback, generates for many systems a limit cycle. A describing function analysis shows that the frequency of the oscillation is the critical frequency (the frequency for which the phase is $-180^{\circ}$ ). Moreover the critical gain of the system can be approximated using the oscillation amplitude. This method has been widely used for process identification and controller tuning due to the simplicity of the real-time implementation as well as the acceptance of the approximative describing function method. Relay feedback can be applied to closed-loop systems too. In [10] two closed-loop relay tests are proposed to estimate the critical and the crossover frequency (the frequency for which the amplitude of the open-loop system is one), as well as the gain and phase margin [9]. This procedure has been generalized in [2] to estimate the frequency at which a user defined transfer function achieves a given magnitude. Among these functions, one can choose between the loop transfer function and the sensitivity function. No method is however proposed for determining the infinity norm of the sensitivity function. In [3] a stability analysis of the limit cycles is provided for the experiment proposed in [2]. This analysis considers that the system is perfectly known, which is generally not the case in practice.

The inverse of the infinity norm of the sensitivity function, which is simply the shortest distance from the Nyquist curve to the critical point -1 , constitutes particularly an important robustness margin and gives very significant informations about the closed-loop system. It quantifies how sensitive is the closed-loop system to variations of the considered plant, and can be seen as a better robustness indicator than the phase and the gain margins. On the other hand the sensitivity function is closely related to the disturbance amplification. When designing a controller, it can be important to control the maximal amplification of disturbances. This can be done by limiting the largest magnitude of the sensitivity function.

Various methods for measuring the infinity norm of the sensitivity function exist nowadays. However, they do not give always complete satisfaction, due either to their complexity or to their measuring accuracy. To illustrate this fact, consider the non-parametric identification methods: First of all a signal containing interesting frequencies must be chosen as the reference of the closed-loop system. The sensitivity function at the considered frequencies is given by the ratio of the Fourier transforms of the error and the reference signals (or respectively by the ratio of the cross spectrum between the error and the ref- 
erence signal over the spectrum of the reference signal). In practice the Fourier transforms are computed numerically with truncated signals in the time domain, which causes in particular a local averaging of the resulting function in the frequency domain. If the amplitude of the sensitivity function has a peak at its maximal value, a loss of resolution of the identified function will be specially noticeable in this frequency region. For this reason, this method is not always adapted for measuring the infinity norm of the sensitivity functions. Model-based identification methods in the time domain give on the other hand good results, but suffer from their complexity. Moreover these methods require a priori informations of the plant.

In this paper a nonlinear scheme is proposed to measure the frequency at which the magnitude of the sensitivity function has a desired value. The main property of this scheme is that it becomes passive if the desired value is chosen larger than the infinity norm of the sensitivity function. This property together with the limit cycle stability analysis given by the wellknown describing function method leads to a simple procedure for measuring the infinity norm of the sensitivity function. Simulation results show the effectiveness of the proposed method.

The paper is organized as follows: In Section 2, a nonlinear scheme is presented and the properties of the generated limit cycles are discussed. A passivity analysis, as well as limit cycles stability considerations are provided in Section 3. A method for measuring the infinity norm of the sensitivity functions is then proposed in Section 4 and illustrated with an example. Simulation results are given in Section 5. Finally, some concluding remarks are made in Section 6.

\section{Closed-Loop Scheme and Limit Cycles Char- acteristics}

In this section, the scheme of Fig. 1 is considered and the characteristics of the generated limit cycles are studied. $G(s)$ is an unknown linear time-invariant transfer function, $K(s)$ the transfer function of a controller that stabilizes the closed-loop system:

$$
G_{c l}(s)=\frac{K(s) G(s)}{1+K(s) G(s)}
$$

$k$ is the slope of the saturation nonlinearity, and $\alpha$ a positive real number. A basic requirement is that the closed-loop transfer function

$$
\frac{Y(s)}{Y_{s}(s)}=\frac{\frac{\alpha}{\alpha+1} K(s) G(s)}{1+\frac{\alpha}{\alpha+1} K(s) G(s)}
$$

is stable. This is, however, the case in general when $K(s) G(s)$ is stable (since the loop gain of $G_{c l}(s)$ is reduced by a factor of $\left.\frac{\alpha}{\alpha+1}<1\right)$.

This scheme is similar to the one proposed in [2] to generate limit cycles for the measurement of several points of the sensitivity function. One of the differences is the use of a saturation nonlinearity in the scheme. This change brings two new important advantages:

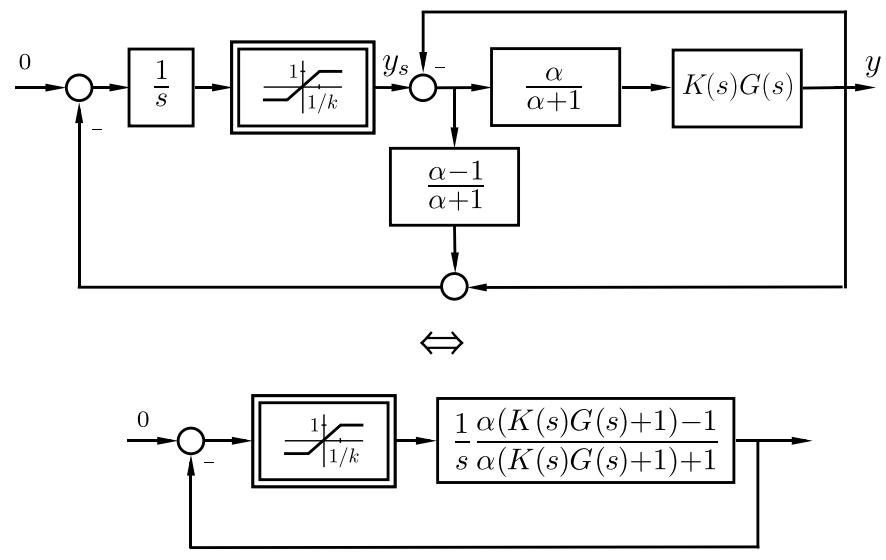

Figure 1: Nonlinear feedback structure

1. By adjusting the slope $k$, the describing function approach can be made more accurate. Thus the measurement precision can be improved (see Section 4).

2. If $\alpha$ is chosen larger than the infinity norm of the sensitivity function $\gamma=\left\|\frac{1}{1+K(s) G(s)}\right\|_{\infty}$, the scheme becomes asymptotically stable (see Section 3.1).

The method proposed in this paper will benefit of these new properties to measure the infinity norm of the sensitivity function. The saturation level can be set to 1 without loss of generality.

The describing function method will be used hereafter to determine the properties of the limit cycles generated by the considered scheme, as well as the necessary condition for their occurrence. This method is an approximative approach. It assumes that a limit cycle occurring at the output of a feedback system with static nonlinearities contains only the first harmonic. Higher harmonics are attenuated by the process. The nonlinearities can then be described by simple nonlinear gains.

The condition for obtaining a limit cycle based on the describing function analysis is given by:

$$
\frac{1}{j \omega} \frac{\alpha(K(j \omega) G(j \omega)+1)-1}{\alpha(K(j \omega) G(j \omega)+1)+1}=-\frac{1}{N_{s}(a, k)}
$$

where $a$ is the amplitude of the signal at the input of the saturation nonlinearity, and $N_{s}(a, k)$ is the describing function of the latter:

$N_{s}(a, k)=\frac{2 k}{\pi}\left[\arcsin \left(\frac{1}{k a}\right)+\frac{1}{k a} \sqrt{1-\frac{1}{k^{2} a^{2}}}\right] \in[0, k]$

To simplify the notation in the sequel, let us define the function seen by the saturation as:

$$
F_{\alpha}(j \omega)=\frac{1}{j \omega} \frac{\alpha(K(j \omega) G(j \omega)+1)-1}{\alpha(K(j \omega) G(j \omega)+1)+1}
$$

Thus an oscillation may occur if the Nyquist curve of $F_{\alpha}(j \omega)$ intersects the half straight line $\left(-\infty,-\frac{1}{k}\right]$. Eq. 3 is 


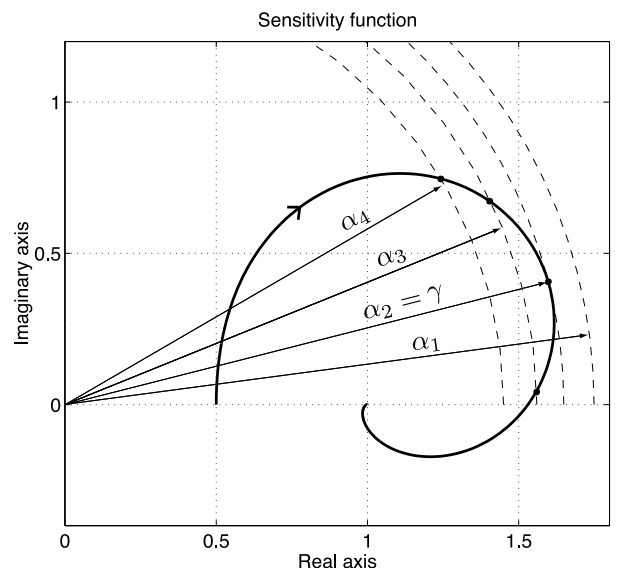

Figure 2: A typical sensitivity function

equivalent to

$$
\frac{1}{j \omega} \frac{1-\frac{1}{\alpha(K(j \omega) G(j \omega)+1)}}{1+\frac{1}{\alpha(K(j \omega) G(j \omega)+1)}}=-\frac{1}{N_{s}(a, k)}
$$

which gives

$$
\frac{1}{1+K(j \omega) G(j \omega)}=\alpha\left[\frac{-1-j \omega \frac{1}{N_{s}(a, k)}}{-1+j \omega \frac{1}{N_{s}(a, k)}}\right]
$$

Since $N_{s}(a, k)$ is a real positive function, the term on the right hand side of Eq. (7) has a modulus of $\alpha$. The presence of a limit cycle at the frequency $\omega_{i}$ implies

$$
\left|\frac{1}{1+K\left(j \omega_{i}\right) G\left(j \omega_{i}\right)}\right|=\alpha
$$

Moreover

$$
\operatorname{Im}\left\{\frac{1}{1+K\left(j \omega_{i}\right) G\left(j \omega_{i}\right)}\right\} \geq 0
$$

This property follows straightforwardly from Eq. 7. Thus, an oscillation may occur if there exists an intersection between the frequency response of the sensitivity function in the complex plane and the half-circle located in the upper half-plane with the radius $\alpha$. Moreover, the frequency of the oscillation is the frequency at which the amplitude of the sensitivity function is $\alpha$.

Fig. 2 depicts the Nyquist plot of a typical sensitivity function and half-circles with different radius. In the case where $\alpha$ is smaller then $\gamma$, there can be one $\left(\alpha=\alpha_{4}\right)$ or more $\left(\alpha=\alpha_{3}\right)$ intersections between the half-circle and the curve of the sensitivity function. Limit cycles may occur in these cases, however they may be stable or unstable. A discussion on the limit cycle stability will show in the next section which intersections correspond to stable or unstable limit cycles.

\section{Stability Analysis}

This section is devoted to a stability analysis of the system and the limit cycles that where discussed in the previous section.
If $\alpha$ is chosen larger than the infinity norm of the sensitivity function $\gamma$ (for example $\alpha=\alpha_{1}$ in Fig. 2), no intersection exists between the half-circle with the radius $\alpha$ and the sensitivity function. In this way the system will not enter a limit cycle, and is asymptotically stable. This affirmation rises from the preceding describing function analysis, but will also be proven in a more rigorous way hereafter through a passivity analysis. If $\alpha$ is smaller then $\gamma$, sufficient conditions for the presence of limit cycles will then be discussed. In particular the effect of the saturation slope $k$ will be analyzed. Based on this analysis, a method will be given to tune $\alpha$ and $k$ in order to identify the largest magnitude of the sensitivity function with an appropriate accuracy.

\subsection{Passivity Analysis}

Consider the scheme of Fig. 3. This scheme is equivalent to the one shown in Fig. 1. This closed-loop system can be di-

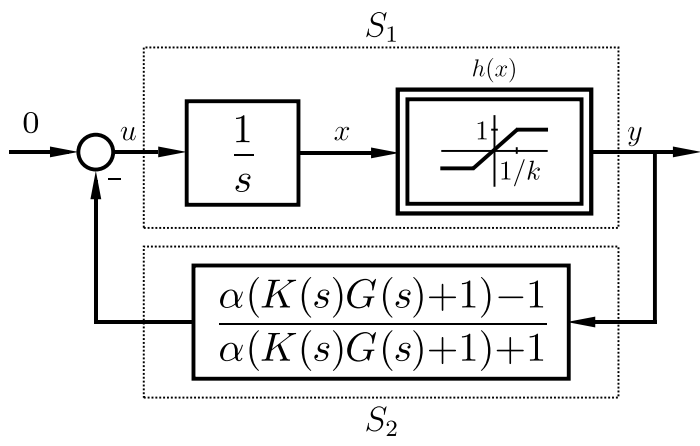

Figure 3: Equivalent scheme for passivity analysis

vided into two subsystems: $S_{1}$ and $S_{2} . S_{1}$, is composed of the integrator and the saturation, and

$$
S_{2}=s F_{\alpha}(s)=\frac{\alpha(K(s) G(s)+1)-1}{\alpha(K(s) G(s)+1)+1}
$$

If one of these subsystems is passive, and the other one strictly passive, then the well-known passivity theorem demonstrates the strictly passivity of the whole system [4].

To demonstrate the passivity of $S_{1}$, consider this subsystem in its state space representation:

$$
\begin{aligned}
& \dot{x}=u \\
& y=h(x)
\end{aligned}
$$

where $u$ is the system input, $y$ the output and $x$ describes the state of the system. The function $h(x)$ represents the saturation nonlinearity. According to the definition of passivity, the system $S_{1}$ is passive (but not strictly), if there exists a lower bounded positive function $V$, such that $\dot{V}=y u$. Since the function $V=\int_{0}^{x} h(\sigma) d \sigma$ verifies these conditions for all $x$ :

$$
\begin{aligned}
V & =\int_{0}^{x} h(\sigma) d \sigma>0 \\
\dot{V} & =\frac{d}{d t} \int_{0}^{x} h(\sigma) d \sigma=h(x) \dot{x}=y u
\end{aligned}
$$


the system $S_{1}$ is passive.

The strictly passivity of the system $S_{2}$ can be demonstrated with the relations between the small-gain and the passivity theorem, which are investigated in [1]. These relations show that, for a transfer function $E(s)$ :

$$
\text { If } \begin{aligned}
& \|E(s)\|_{\infty}<1 \\
& \quad \Rightarrow(I-E(s))(I+E(s))^{-1} \text { is strictly passive }
\end{aligned}
$$

By substituting $E(s)$ with $\frac{1}{\alpha(1+K(s) G(s))}$, the above affirmation becomes:

$$
\text { If } \begin{array}{r}
\left\|\frac{1}{(1+K(s) G(s))}\right\|_{\infty}<\alpha \\
\Rightarrow \frac{\alpha(K(s) G(s)+1)-1}{\alpha(K(s) G(s)+1)+1} \text { is strictly passive }
\end{array}
$$

It follows that the subsystem $S_{2}$ and thus also the whole system of Fig. 3 are strictly passive if $\alpha>\gamma$. Note that the whole system still remains passive if the saturation is replaced with any static nonlinearity that is of the same sign as its argument, although not necessarily continuous. This is however not the case if the saturation is replaced with an ideal relay: Sliding modes oscillations will occur.

\subsection{Stability and Occurrence of Limit Cycles}

In Section 2 the necessary condition for the occurrence of limit cycles has been given, which is the existence of one or more intersections between the Nyquist plot of the sensitivity function and the half-circle with the radius $\alpha$ located in the upper half-plane, and centered at the origin. In this chapter, sufficient conditions will be treated. This study will be done in two steps. First, a stability analysis of limit cycles will be provided. Then, the existence of a trivial solution, which consist of a stable equilibrium point, will be studied.

Consider the case where $\alpha<\gamma$. Eq. 3 and Eq. 7 imply that the number $n$ of intersections between the Nyquist curve of the sensitivity function and the half-circle of radius $\alpha$ corresponds to the number of intersections between the negative real axis and the transfer function $F_{\alpha}(j \omega)$. The latter are denoted with $P_{1}, P_{2}, \ldots, P_{n}$ in the ascending order of the corresponding frequencies ( $P_{1}$ corresponds to the lowest frequency and $P_{n}$ to the largest). Since the Nyquist curve of the sensitivity function intersects successively the half-circle, the points $P_{1}, \ldots, P_{n}$ are placed respectively in an ascending order on the real axis. Moreover, the function $F_{\alpha}(j \omega)$ has the following boundary conditions, due to the presence of the integrator:

$$
\lim _{\omega \rightarrow 0} F_{\alpha}(j \omega)=-j \infty, \quad \lim _{\omega \rightarrow \infty} F_{\alpha}(j \omega)=0
$$

In Fig. 4, an example for the function $F_{\alpha}(j \omega)$ is depicted. At least one intersection exists between $F_{\alpha}(j \omega)$ and the half straight line $\left(-\infty,-\frac{1}{k}\right] \in(-\infty, 0]$, if $k>-\frac{1}{P_{1}}$, where $k$ is the slope of the saturation. To each intersection corresponds a stable or unstable limit cycle. Furthermore, the limit cycle

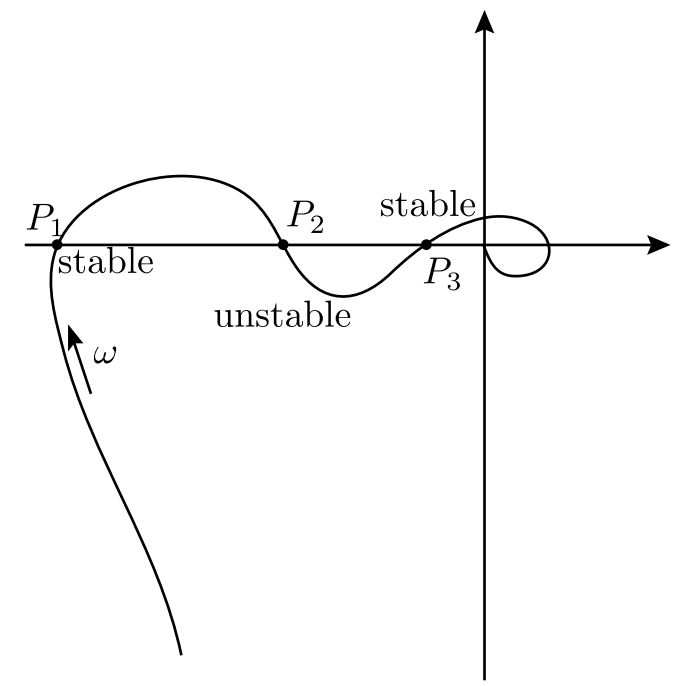

Figure 4: $F_{\alpha}(j \omega)$

stability criterion, based on the extended Nyquist criterion [4], shows that stable and unstable limit cycles occur alternatively. Moreover, the limit cycle which corresponds to the first intersection $P_{1}$ is always stable. Thus, if $k \in\left[-\frac{1}{P_{1}} ;+\infty\right)$, at least one stable limit cycle exists. The existence of a stable limit cycle does however not necessarily impose its occurrence. A stable limit cycle will occur if it is the only stable solution of the system. Otherwise it may occur or not, depending on the initial conditions of the system.

Let us now consider the stability of the equilibrium point 0 (input, output and all states of the system are 0), that may also be a solution of the closed-loop system. For this purpose, consider the system of Fig. 1 at the point 0 and infinitesimal disturbances entering somewhere in the loop. If 0 is an stable equilibrium point, the disturbances in the loop will be damped. Since the output signal of the saturation (which is $<<1$ ) is not saturated, the closed-loop system is linear in this region. Its open-loop transfer function is:

$$
k F_{\alpha}(s)=k \frac{1}{s} \frac{\alpha(K(s) G(s)+1)-1}{\alpha(K(s) G(s)+1)+1} .
$$

Moreover $F_{\alpha}(s)$ does not have unstable poles, because the poles of $s F_{\alpha}(s)$ are those of the closed loop system of Eq. 2, which is stable according to the basic assumption of Section 2. Hence, stability can be determined with the Nyquist criterion as followed: The closed-loop system is stable if and only if $F_{\alpha}(j \omega)$ doesn't encircle the point $-\frac{1}{k}$. According to the characteristics of the curve $F_{\alpha}(j \omega)$, it follows that the linear system is stable only if the index $m$ of the intersection point $P_{m}$, which is directly smaller than $-\frac{1}{k}$, is even.

To clarify these affirmations, let us consider the example of Fig. 4. As long as $-\frac{1}{k}>P_{1}$, at least one stable limit cycle exists in the system. If $P_{1}<-\frac{1}{k}<P_{2}$ or $-\frac{1}{k}>P_{3}$ limit cycles are the only stable solutions of the system. On the other hand, if $P_{2}<-\frac{1}{k}<P_{3}, 0$ is a stable equilibrium point, and the system is also locally asymptotically stable. 


\section{Measuring the Infinity Norm of the Sensitivity Function}

Based on the preceding stability analysis, as well as the passivity property, a method with sufficient accuracy is proposed in this section to identify the infinity norm of the sensitivity function of systems. The procedure is illustrated with the following example:

$$
K(s) G(s)=\frac{1}{(s+1)^{5}}
$$

for which the infinity norm of the sensitivity function $\gamma$ is 1.65 . The Nyquist plot of its sensitivity function is depicted in Fig. 6.

The proposed method consists of the following steps:

1. Choose a large value for the saturation slope $k$, so that the saturation can be regarded as a non-ideal relay, and $\alpha$ large enough to obtain a passive system $(\alpha>\gamma)$. Fig. 5a shows $F_{\alpha_{1}=1.7}(j \omega)$ for the considered example.

2. Reduce then gradually $\alpha$ until the occurrence of a limit cycle.

When $\alpha$ becomes smaller than $\gamma$, a stable limit cycle exists, but will not occur as long as the point 0 is an stable equilibrium point. This is the case, if the number of intersections between the half-straight line $\left(-\infty,-\frac{1}{k}\right]$ $(\approx(-\infty, 0])$ and the Function $F_{\alpha}(j \omega)$ is even (See Fig. $5 \mathrm{~b}$ for $\left.\alpha_{2}=1.6\right)$. This number corresponds however to that of the intersections between the frequency response of the sensitivity function and the half-circle of radius $\alpha$ centered at the origin (see Fig. 6). The number of intersections between the two curves is 2 if $\alpha_{u}<\alpha<\gamma$, where $\alpha_{u}$ is the magnitude of the sensitivity function at $\omega_{u}$. For $\frac{1}{1+K(0) G(0)}<\alpha<\alpha_{u}$ there is one intersection. Thus a limit cycle occurs only when $\alpha$ reaches $\alpha_{u}$ (see Fig. 5c, $\left.\alpha_{3}=\alpha_{u}=1.53\right)$.

3. Identify the intersection point $P_{1}\left(\alpha_{u}\right)$ between $F_{\alpha_{u}}(j \omega)$ and the negative real axis through the limit cycle amplitude obtained in the experiment shown in Fig. 1 according to Eq. 3 and Eq. 4.

4. Choose $k=k_{1}>-\frac{1}{P_{1}\left(\alpha_{u}\right)}$. For this value the limit cycle will not be damped. Proposed value: $k_{1}=-\frac{1}{4 P_{1}\left(\alpha_{u}\right)}$ (Fig.5c).

In fact, $k$ is a determining factor for the accuracy of the measurements. If $k$ is chosen very large (relay), the output signal of the saturation will be a square wave. The presence in this signal of high harmonics that cannot be attenuated efficiently by the function $F_{\alpha}(j \omega)$, due to its relative degree of 1 , will lead to significant identification errors. If $k$ is reduced, the output of the saturation will contain less harmonics, improving the precision of the estimates. However, a small saturation level ensures a minimum distance between $-1 / k$ and $P_{1}(\alpha)$ which guaranties the continuity of the limit cycle. The proposed value for $k$ is a good compromise between harmonic elimination and limit cycle conservation.
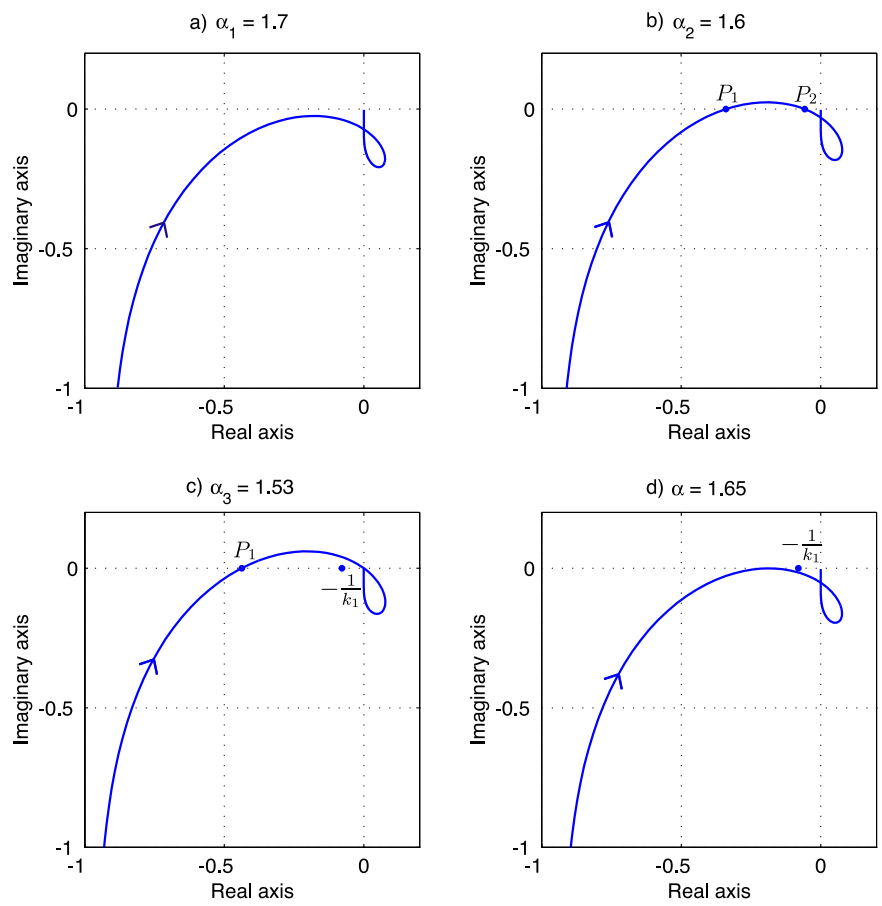

Figure 5: $F_{\alpha}(j \omega)$ for different values of $\alpha$

5. Increase slowly $\alpha$ until the limit cycle starts damping. This value gives the infinity norm of the sensitivity function (see Fig. 5d).

During this step, if the saturation level becomes too small for a non-damped limit cycle, the slope of saturation $k$ should be increased. However for the systems presented in Section 5 the saturation slope did not need to be readjusted during the experiment.

Note at this point that the experiment duration is usually reasonable. The only part of the procedure which is time consuming is Step 5, where $\alpha$ should be increased slowly from $\alpha_{u}$ to $\gamma$ for a good measurement. However $\alpha_{u}$ is close to $\gamma$ for many systems.

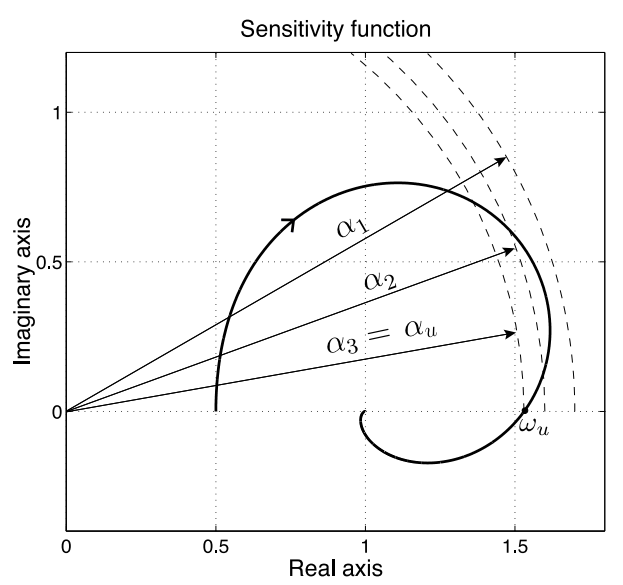

Figure 6: Sensitivity function 


\begin{tabular}{|c|c|c|c||c|c|c||c|c|c|}
\hline$K(j \omega) G(j \omega)$ & $\gamma_{e}$ & $\gamma_{m}$ & $\operatorname{Err}(\%)$ & $\omega_{\infty_{e}}$ & $\omega_{\infty_{m}}$ & $\operatorname{Err}(\%)$ & $G_{m_{e}}$ & $G_{m_{m}}$ & $\operatorname{Err}(\%)$ \\
\hline$\frac{1}{(s+1)^{3}}$ & 1.28 & 1.28 & - & 1.12 & 1.13 & 0.9 & 8 & 7.67 & 4.3 \\
\hline$\frac{1}{(s+1)^{5}}$ & 1.65 & 1.61 & 2.4 & 0.631 & 0.628 & 0.5 & 2.89 & 3 & 3.8 \\
\hline$\frac{0.5}{s(s+1)^{2}}$ & 1.84 & 1.84 & - & 0.601 & 0.607 & 0.99 & 4 & 4.03 & 0.75 \\
\hline$\frac{0.5 s+1}{s(0.2 s+1)(s+1)} e^{-0.5 s}$ & 2.01 & 2.00 & 0.5 & 1.15 & 1.17 & 1.7 & 2.61 & 2.88 & 10.5 \\
\hline
\end{tabular}

Figure 7: Simulation results

Notice also that $\alpha_{u}$ is equal to $\frac{G_{m}}{G_{m}-1}$, where $G_{m}$ is the gain margin of $K(j \omega) G(j \omega)$ defined as:

$$
G_{m}=-\frac{1}{K\left(j \omega_{u}\right) G\left(j \omega_{u}\right)}
$$

where the critical frequency $\omega_{u}$ is the frequency where $\angle K(j \omega) G(j \omega)=-\pi$. The phase of the sensitivity function at this frequency $\angle \frac{1}{K\left(j \omega_{u}\right) G\left(j \omega_{u}\right)+1}$ is obviously zero. The experiment provides also the value of the gain margin at no extra cost. The method is restricted to system for which the frequency of the sensitivity infinity norm is smaller than the critical frequency. However this constitutes a large class of plants encountered in practice.

\section{Simulation Results}

The proposed method has been tested by simulation on different systems. The results and comparisons with exact values are shown in Fig 7. $\gamma_{e}$ and $\gamma_{m}$ are the exact and the measured values of the infinity norm of the sensitivity function, respectively. $\omega_{\infty_{e}}$ and $\omega_{\infty_{m}}$ are the exact and measured values of the corresponding frequency. $G_{m_{e}}$ and $G_{m_{m}}$ are the exact and measured gain margins. Small relative errors $(\operatorname{Err}(\%))$ between exact and measured values show the accuracy of the proposed method.

\section{Conclusion}

A feedback structure containing a saturation nonlinearity has been proposed for infinity norm measurement of sensitivity function. It has been shown that the scheme may produce limit cycles at a frequency where the sensitivity function achieves a selected magnitude. The use of a saturation nonlinearity instead of a relay brings following improvements to the scheme: On the one hand a judicious choice of the saturation slope can improve in a significant way the measurement accuracy. On the other hand a passivity property is added for the case where the magnitude is chosen larger than the sensitivity infinity norm. Stability analysis have been carried out to show the sufficient conditions for the occurrence of limit cycles. Based on these considerations, a simple procedure has been proposed to measure the infinity norm of the sensitivity function for a large class of practical systems. Simulation results show the effectiveness of the proposed method. This method can be used in the identification step of an auto-tuning procedure.

\section{Acknowledgements}

This research work is financially supported by the Swiss National Science Foundation under grant No. 2100-064931.01

\section{References}

[1] B. D. O. Anderson. The small-gain theorem, the passivity theorem and their equivalence. Journal of The Franklin Institute, 293(2):105-115, 1972.

[2] G. H. M. de Arruda and P. R. Barros. Relay based closed loop transfer function estimation. In $A C C$, pages 1812 1816, June 2000.

[3] G. H. M. de Arruda, P. R. Barros, and A. S. Bazanella. Dynamics of a relay based frequency response esitmator. In 15th Triennial World Congress of IFAC, June 2002.

[4] J-J E. Slotine and W. Li. Applied Nonlinear Control. Prentice-Hall International, 1991.

[5] K. J. Aström and T. Hägglund. Automatic tuning of simple regulators with specifications on phase and amplitude margins. Automatica, 20(5):645-651, 1984.

[6] A. Karimi, D. Garcia, and R. Longchamp. PID controller tuning using bode's integrals. Transaction on Control Systems Technology, accepted for publication.

[7] A. Karimi, D. Garcia, and R. Longchamp. Iterative controller tuning using Bode's integrals. In CDC, FrM07-6, pages 4227-4232, December 2002.

[8] A. Karimi, D. Garcia, and R. Longchamp. PID controller design using Bode's integrals. In ACC, FP13-6, pages 5007-5012, May 2002.

[9] R. Longchamp and Y. Piguet. Closed-loop estimation of robustness margins by the relay method. IEEE ACC, pages 2687-2691, 1995.

[10] T. S. Schei. Closed-loop tuning of PID controllers. In ACC, FA12, pages 2971-2975, 1992. 